\title{
Crocin induces autophagic cell death and inhibits cell invasion of cervical cancer SiHa cells through activation of PI3K/AKT
}

\author{
Jian Zhang ${ }^{1 \#}$, Shaoping Yang ${ }^{1 \#}$, Kana Wang $^{2}$, Yu Huang ${ }^{1}$, Nian Yang $^{1}$, Zhongmei Yang $^{1}$, Zhenrong Zheng $^{1 \wedge}$, \\ Yujue Wang ${ }^{1} \wedge$ \\ ${ }^{1}$ Department of Obstetrics and Gynecology, Sichuan Academy of Medical Sciences \& Sichuan Provincial People’s Hospital, Chengdu, China; \\ ${ }^{2}$ Department of Obstetrics and Gynecology, West China Second Hospital, Sichuan University, Chengdu, China \\ Contributions: (I) Conception and design: Z Zheng, Y Wang; (II) Administrative support: K Wang, Y Huang, N Yang; (III) Provision of study \\ materials or patients: J Zhang, S Yang; (IV) Collection and assembly of data: J Zhang, S Yang; (V) Data analysis and interpretation: Z Yang; (VI) \\ Manuscript writing: All authors; (VII) Final approval of manuscript: All authors. \\ \#These authors contributed equally to this work and are co-first authors. \\ Correspondence to: Zhenrong Zheng; Yujue Wang. No. 32, West Second Section, First Ring Road, Chengdu, China. Email: 675318179@qq.com; \\ wangyjuea@126.com.
}

Background: Cervical cancer is a prevalent tumor mainly induced by Human Papilloma Virus (HPV). Autophagy was inactivated with HPV to promote cancer progression. Here we explored the effects of crocin on cervical cancer cells, mainly on autophagy and apoptosis.

Methods: SiHa cells were treated with crocin, and proliferation, metastases, apoptosis and autophagy were measured using a CCK-8 assay, transwell migration assay, flow cytometry and immunofluorescence. Protein levels were measured using western blotting. The antitumor effects of crocin were validated in female BALB/ c nude mice injected with SiHa cells.

Results: The result showed that 2, 4, 8 and $16 \mathrm{mM}$ of crocin significantly reduced the viability of SiHa cells within $24 \mathrm{~h}$. Subsequently, 0, 1, 2 and $4 \mathrm{mM}$ crocin concentrations were used in later experiments. Treatment with crocin reduced invasive cells, while increasing autophagic and apoptotic cells dose-dependently. The enhanced apoptosis and autophagy were partly validated by an increase in cleaved caspase-3/caspase-3, cleaved caspase-9/caspase9, LC3B II/I, Beclin1 and ATG7. AMPK and mTOR were inactivated with crocin treatment, while PI3K was activated. These results indicated that crocin might promote autophagy and apoptosis by inactivating AMPK and mTOR signaling. Tumor progression was inhibited in mice treated with $50 \mathrm{mg} / \mathrm{kg} / \mathrm{d}$ of crocin, which was demonstrated by smaller tumor volumes, less VEGF expression, more intense caspase-3 staining and increased LC3B II/I in the tumor tissues.

Conclusions: Crocin inhibited the progression of cervical cancer in vitro and in vivo, possibly through inactivation of AMPK and mTOR, inhibition of proliferation and invasion, and promotion of autophagy and apoptosis. These results support the potential therapeutic value of crocin in treating cervical cancer.

Keywords: Crocin; cervical cancer; autophagy; invasion

Submitted Jul 29, 2020. Accepted for publication Sep 04, 2020.

doi: $10.21037 / \mathrm{atm}-20-5882$

View this article at: http://dx.doi.org/10.21037/atm-20-5882

^ ORCID: Zhenrong Zheng, 0000-0002-4586-6268; Yujue Wang, 0000-0002-4223-6842. 


\section{Introduction}

Cervical cancer, the most common human papillomavirus (HPV)-associated cancer, is one of the most prevalent tumors among women in the developing world (1). As vaccination is still largely economically unavailable, cervical cancer continues to be a growing major public health issue (2). Once diagnosed, the prognosis of cervical cancer is poor, especially in women with metastatic or recurrent disease. Novel therapeutic approaches including immunotherapy and targeted therapy, for example platinum combined with bevacizumab or pembrolizumab, have been able to extend overall survival (3). However, these treatment approaches remain insufficient and expensive.

Autophagy have critical effects on HPV infection and development of cervical cancer [reviewed in (4)]. HPV inhibited autophagy via inhibiting the fusion of autophagosome-lysosome with multiple ways. Autophagy was inhibited upon infection to avoid digestion, and in later processes to promote proliferation and carcinogenesis of infected cells. Inhibited autophagy could also avoid apoptotic cell death induced with violent autophagy. It is thus very important to restore the autophagic processes in treating cervical cancer.

Saffron, a traditional Chinese medicine, is the dried dark-red stigma of the plant Crocus sativus L. Saffron has been used traditionally to treat some conditions (5), such as certain cancers, neurological defects, cognitive problems and inflammatory diseases. One of the main bioactive components of Saffron is crocin, a soluble carotenoid with two D-gentiobioside glycosyl esters. The mechanism of action of crocin is complex (5), and includes the inhibition of DNA and RNA synthesis, reversion of epithelialmesenchymal transition (EMT), suppression of cell invasion and metastasis, targeting of cellular topoisomerase and microtubules, induction of apoptosis, suppression of telomerase activity, reduction of oxidative stress, and epigenetic effects. These mechanisms suggest that crocin may have potential as an antitumor agent. Furthermore, emerging evidence suggests that saffron is selectively toxic to tumor cells but safe to normal cells $(6,7)$. Crocin was implied to induce autophagy and promote apoptosis in hepatocellular carcinoma by inhibiting Akt/mTOR activity (8). Crocin has also demonstrated time and dose dependent inhibition of cervical cancer cell viability, and induced apoptosis and cell cycle arrest (7). However, whether or not autophagy and apoptosis could be induced with crocin in cervical cancer still require further investigation.

To this end, the current study tested the effects of crocin on SiHa cells. Viability, apoptosis, autophagy and potential signaling molecules were investigated, and also validated in female BALB/c nude mice. We present the following article in accordance with the ARRIVE reporting checklist (available at http://dx.doi.org/10.21037/atm-20-5882).

\section{Methods}

\section{Cell culture and viability}

SiHa cells were purchased from the American Type Culture Collection (Rockville, MD, USA), and were maintained at $37{ }^{\circ} \mathrm{C}$ with $5 \% \mathrm{CO}_{2}$. Cells were cultured with Dulbecco's modified eagle medium (DMEM) supplemented with $10 \%$ fetal bovine serum and $1 \%$ penicillin/streptomycin solution. For testing cell viability, the $\mathrm{SiHa}$ cells were seeded onto 96-well plates with $3 \times 10^{3}$ cells/well in $100 \mu \mathrm{L}$ of DMEM medium supplemented with $0.06,0.13,0.25,0.5,1,2,4,8$ and $16 \mathrm{mM}$ of crocin (\#17304, Sigma-Aldrich, Missouri, USA). After 22 hours, $10 \mu \mathrm{L}$ CCK-8 solution was dissolved in $90 \mu \mathrm{L}$ of DMEM medium and subsequently added to each well. The plates were incubated for 2 hours, and the absorbance value was measured at $450 \mathrm{~nm}$.

\section{Transwell migration assay}

A transwell migration assay was conducted to measure cell invasion. The Matrigel (Becton, Dickinson and Company, Bioscience, San Jose, CA, USA) was pre-cooled at $4{ }^{\circ} \mathrm{C}$ overnight for liquidation, and diluted with serum-free medium on ice for a 1:1 dilution. The Matrigel mixture $(15 \mu \mathrm{L})$ was then added to the upper chamber (Costar, Cambridge, MA, USA) in each well and cultured at $37^{\circ} \mathrm{C}$ for 1 hour, then rinsed three times using serum-free medium. SiHa cells $\left(1 \times 10^{5}\right.$ cells) suspended in DMEM were added to the upper chamber, and $600 \mu \mathrm{L}$ DMEM complete medium containing $15 \%$ FBS was added to the basolateral chamber. To both chambers, $0,1,2$ or $4 \mathrm{mM}$ of crocin was added, then cultured for 24 hours. The chambers were then fixed with $50 \%$ methyl alcohol for 15 minutes. Crystal violet staining was performed, and 6 visual fields from each well were randomly observed and imaged under an inverted microscope (200×, Olympus Optical Co., Ltd.). The average number of cells was calculated in each field. 


\section{Flow cytometry}

To detect cell apoptosis, $\mathrm{SiHa}$ cells were treated with crocin $(0,1,2$ and $4 \mathrm{mM})$ for 24 hours then digested with trypsin. The trypsin digested cells $\left(1 \times 10^{6}\right)$ were subjected to flow cytometry using an Annexin V-FITC/PI Apoptosis Detection kit (Beyotime, Shanghai, Chain) following the manufacturer's protocol. Cells with Annexin V-positive values were considered early apoptotic cells, those with PIpositive values were considered necrotic, and those with double positive values were considered late apoptotic.

\section{Western blotting analysis}

Western blotting was performed to measure protein expression. SiHa cells were treated with crocin $(0,1,2$ and $4 \mathrm{mM}$ ) for 24 hours then washed twice with cold PBS, and RIPA lysis buffer (Beyotime) was added to collect protein lysates. Total protein concentration was measured using the BCA Protein Assay Reagent (Pierce, Rockford, IL, USA), and the sample concentrations were diluted with PBS. Equal amounts of protein from experimental groups were subjected to SDS-PAGE and transferred to PVDF membranes. The membranes were blocked with $5 \%$ fat-free milk, probed with the primary and secondary antibodies, then enhanced with a chemiluminescence (ECL) reagent (Amersham Pharmacia Biotech). The following antibodies (Abcam, Cambridge, UK) were used: anti-Caspase-3 (ab32351), anti-Caspase-9 (ab202068), anti-LC3B (ab48394), anti-Beclin1 (ab2 10498), anti-ATG-7 (ab52472), anti-AMPK (ab80039), anti-p-AMPK (ab133448), antiAKT (ab8805), anti-p-AKT (phospho T308, ab38449), anti-mTOR (ab2732), anti-p-mTOR (ab109268), antiVEGF (ab150375), anti-Actin (ab8227) and goat-anti-rabbit IgG (ab205718).

\section{Immunofluorescence}

Immunofluorescence of $\mathrm{SiHa}$ cells was performed as previously described (9). After fixation with 4\% paraformaldehyde for $15 \mathrm{~min}$ at $37{ }^{\circ} \mathrm{C}$, SiHa cells were permeabilized with $0.1 \%$ Triton X-100 (Beyotime, China) for $30 \mathrm{~min}$, and blocked with goat serum for $15 \mathrm{~min}$. Cells were incubated with primary LC3 antibody $(1 \mu \mathrm{g} / \mathrm{m}$, Abcam) overnight at $4{ }^{\circ} \mathrm{C}$, and then with AlexaFluor ${ }^{\circledR} 647$ conjugated secondary antibody (\#4414, 1:2,000, CST, USA) at room temperature for $60 \mathrm{~min}$. DAPI (Beyotime) was used to counterstain the cell nuclei. Immunofluorescence images were acquired with confocal microscopy (LSM 510 Meta, Zeiss, Oberkochen, Germany). LC3 puncta were counted from 10 random fields per slide.

\section{Animal experiments}

The study was approved by the Medical Ethics Committee of West China Second Hospital, Sichuan University (No. 2014-2020). All animal experiments were approved by the Institutional Animal Care and Use Committee of Sichuan Academy of Medical Sciences \& Sichuan Provincial People's Hospital, and were performed according to institutional guidelines. Twelve 4-week-old female BALB/c nude mice were purchased from Shanghai SLAC Laboratory Animal Co., Ltd. The mice were housed in 2 polypropylene cages at room temperature with a relative humidity of $60 \pm 5 \%$ and a photoperiod of $12 \mathrm{~h}$ /day. Approximately $1 \times 10^{7} \mathrm{SiHa}$ cells suspended in $100 \mu \mathrm{L}$ of PBS were injected into the right thigh of all mice. After 5 days, the formation of tumors was confirmed, and mice were randomly divided into two groups and administered as: crocin $(50 \mathrm{mg} / \mathrm{kg} / \mathrm{d}$ ) or saline (vehicle) by referring to previous research (10). After 4 weeks, the mice were sacrificed. The tumors were surgically removed, fixed in $10 \%$ formalin, and subjected to routine histological examination.

\section{Immunobistochemical staining}

Caspase-3 and vascular endothelial growth factor (VEGF) protein expression in xenograft tumors was determined by immunohistochemistry (IHC) (11). The primary antibodies used were the same as those used for western blotting. The fixed tumors were embedded in paraffin and $4 \mu \mathrm{m}$ slices were sectioned. The sections were rehydrated and incubated in citrate buffer for $3 \mathrm{~min}$ at $100{ }^{\circ} \mathrm{C}$. The sections were then incubated with primary antibodies against caspase-3 and VEGF overnight at $4{ }^{\circ} \mathrm{C}$. After further incubation with goat anti-rabbit IgG (Boster Biological Technology, Wuhan, China) for $30 \mathrm{~min}$, the sections were stained with diaminobenzidine and photographed under a microscope. Four random and nonoverlapping positively stained microscopic fields at $400 \times$ magnification were examined in each section. The protein expression levels in each section were measured with integral optical density values (IOD) (IOD = sum of four discontinuous visual fields/4) measured with Image-Pro Plus 6.0 software (Media Cybernetics Inc., Maryland, USA). 


\section{Statistical analysis}

All in vitro experiments were repeated three times. Twotailed unpaired Student's $t$-tests and one-way ANOVA (analysis of variance) were used to evaluate the differences between the two groups. GraphPad Prism 6 software (San Diego, CA) was used for the statistical analysis. All quantitative data were presented as mean \pm standard error of mean. $\mathrm{P}<0.05$ was considered statistically significant.

\section{Results}

\section{Crocin inbibited viability and invasion capacity of SiHa cells}

To test the effects of crocin on $\mathrm{SiHa}$ cells, cells were incubated with $0.06,0.13,0.25,0.5,1,2,4,8$ and $16 \mathrm{mM}$ of crocin in $100 \mu \mathrm{L}$ of medium for 24 hours. The viability of $\mathrm{SiHa}$ cells were dose-dependently reduced with crocin, with 2, 4, 6 and $8 \mathrm{mM}$ significantly reducing cell viability (Figure 1A). In later experiments, 0, 1, 2 and $4 \mathrm{mM}$ concentrations of crocin were established to show its underlying mechanism. The invasive cells tested with a transwell migration assay showed a significant reduction in the $2 \mathrm{mM}$ group, and even fewer in $4 \mathrm{mM}$ group (Figure 1B,C). The results demonstrate the inhibition of cell viability and invasion of $\mathrm{SiHa}$ cells treated with crocin.

\section{Crocin promoted apoptosis of SiHa cells}

Apoptosis is a common downstream mechanism of most anticancer therapeutics. In crocin treated $\mathrm{SiHa}$ cells, apoptosis was significantly induced by 2 and $4 \mathrm{mM}$ of crocin (Figure 2A,B). The induced apoptosis was further validated with the increased ratio of cleaved caspase- $3 /$ caspase- 3 and cleaved caspase-9/caspase-9 (Figure 2C,D). The results indicated that apoptosis was also the downstream mechanism of crocin in the suppression of $\mathrm{SiHa}$ cells.

\section{Crocin enbanced autophagy of SiHa cells}

The ratio of LC3II/I, Beclin1 and ATG7 were all markedly increased after 2 and $4 \mathrm{mM}$ of crocin treatment (Figure $3 A, B$ ). The induced autophagy was also validated by the presence of increased LC3 puncta positive cells after 2 and $4 \mathrm{mM}$ of crocin treatment (Figure 3C,D). These results suggest that autophagy was induced dose-dependently after crocin exposure.

\section{Crocin inactivated AMPK/MTOR}

AMPK, AKT and mTOR are implicated in the regulation of autophagy and apoptosis in crocin treated cells (12). As shown in Figure $4 A, B$, the ratio of p-AMPK/AMPK and $\mathrm{p}-\mathrm{mTOR} / \mathrm{mTOR}$ were inhibited dose-dependently, while $\mathrm{p}-\mathrm{AKT} / \mathrm{AKT}$ increased. These results indicate that AMPK and mTOR signaling were potentially involved in the mechanism of action of crocin in suppressing $\mathrm{SiHa}$ cell proliferation.

\section{Crocin suppressed tumor progression in vivo}

In $\mathrm{SiHa}$ cell-bearing mice, $50 \mathrm{mg} / \mathrm{kg} / \mathrm{d}$ of crocin significantly reduced tumor formation (Figure $5 A$ ). The tumor volumes in the crocin group were significantly smaller than the control group (Figure 5B). Proliferation and metastases were inhibited with crocin (Figure 5C), as seen through increased caspase-3 (Figure 5D) and reduced VEGF (Figure 5E) in treated tumors. Autophagy in the tumors was assessed using western blotting (Figure 5F), and demonstrated that the ratio of LC3II/I was significantly elevated in tumors of crocin treated mice (Figure $5 G$ ).

\section{Discussion}

Persistent viral infection of the high-risk HPV subtype is the main cause of cervical cancer worldwide. The risk for cervical cancer remains high in certain countries, despite the increasing availability of vaccination (13). Chemotherapy for late stage cervical cancer mainly includes carboplatin/ paclitaxel or topotecan/paclitaxel combined with bevacizumab $(14,15)$. Development of novel therapeutic strategies is still required, as current treatment options remain limited. In the present study, we showed that crocin inhibited the progression of cervical cancer in vitro and in vivo, as indicated by the inhibition of proliferation and invasion, and the promotion of autophagy and apoptosis in $\mathrm{SiHa}$ cells and in xenograft mice. These effects were at least partly the result of inactivated AMPK/mTOR pathway, supporting the value of autophagy promoting effects in treating cervical cancer. We suggest that crocin is worthy of further study to combat cervical cancer.

Crocin has been implicated in tumor suppression across multiple cancers, through several complex pathways and mechanisms (16). For example, crocin exerts antitumor effects through the activation of p53 and Bax 


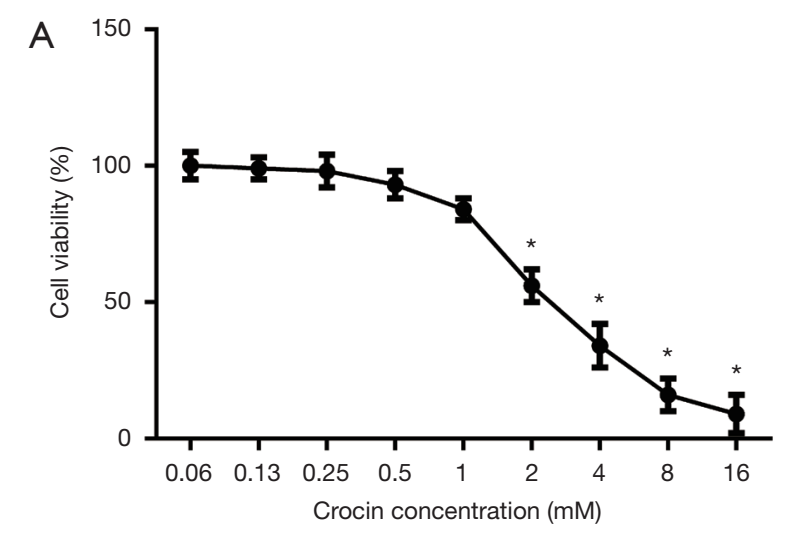

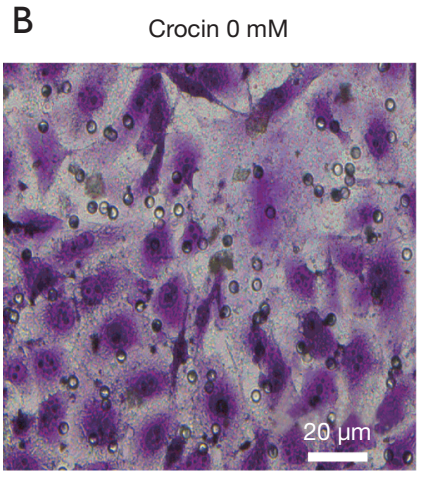

Crocin $2 \mathrm{mM}$

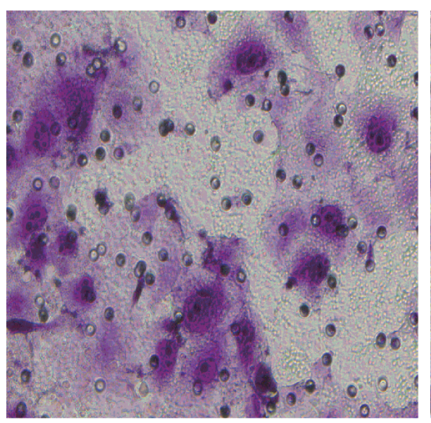

Crocin $1 \mathrm{mM}$

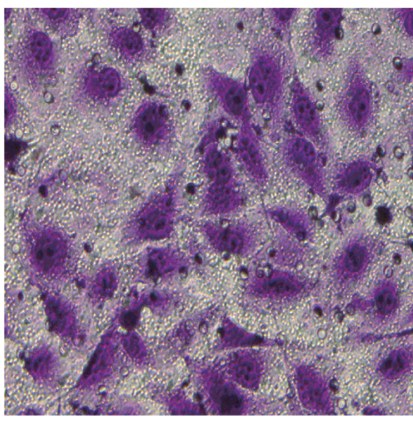

Crocin $4 \mathrm{mM}$

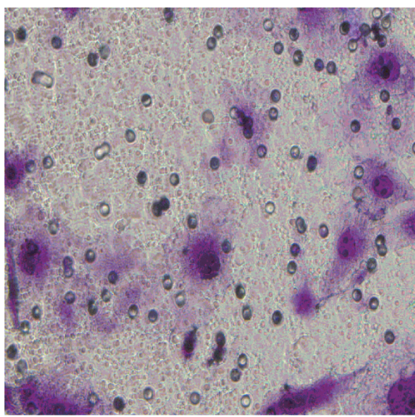

C

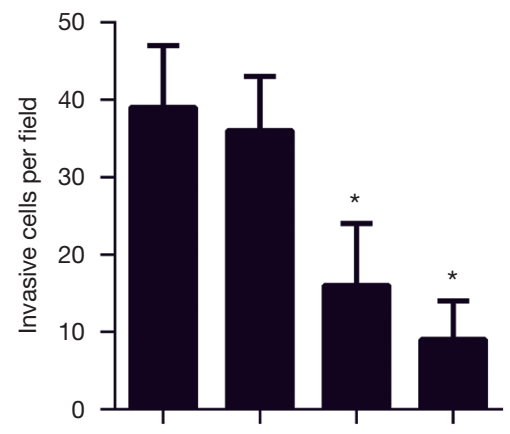

Figure 1 Cell viability and invasion of SiHa cells treated with crocin. (A) SiHa cells were treated with indicated concentrations of crocin for 24 hours and viability was tested using a CCK-8 assay; (B) invasive SiHa cells treated with 0, 1, 2 and 4 mM of crocin for 24 hours and tested with a Matrigel-enabled transwell migration assay, stained with crystal violet and observed under $400 \times$ scope; (C) the invasive cells were counted. *, $\mathrm{P}<0.05$ versus control with $t$-test.

while inactivating Bcl-2 in gastric cancer and lung cancer $(17,18)$, inactivating $\mathrm{NF}-\kappa \mathrm{B}$ and inflammation in colorectal cancer (19), and inhibiting histone deacetylase 2, Wnt/ $\beta$-catenin and CD34 in breast cancer (20-22). Molecular docking has predicted several targets for crocin, including telomeric DNA (23), coronavirus (nCoV2019) main protease (24), human tyrosinase (25), human serum albumin (26), glucagon-like peptide-1 (GLP-1) receptor (27), catalase (28), TLR4 and TGF- $\beta R 1$ (29). Most of these predicted targets have been validated in specific cells. The many predicted targets partly explain the complex mechanism of action for crocin. On the other hand, some of these targets highlight the potential for adverse effects and interactions with other therapeutics that may limit 

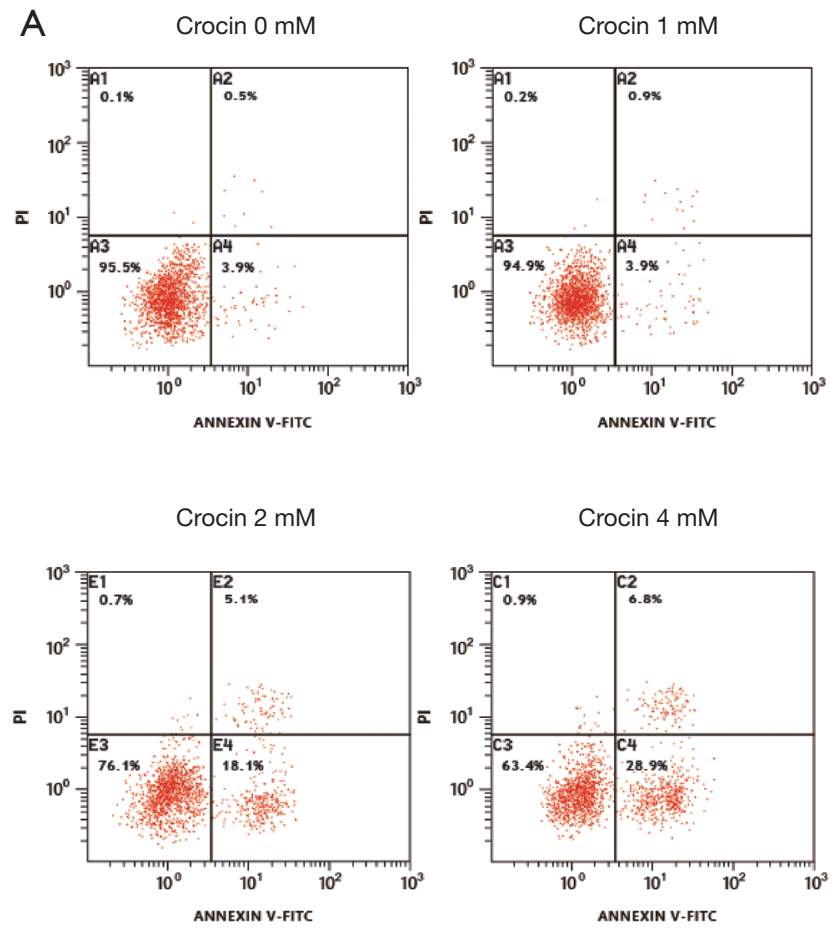

C

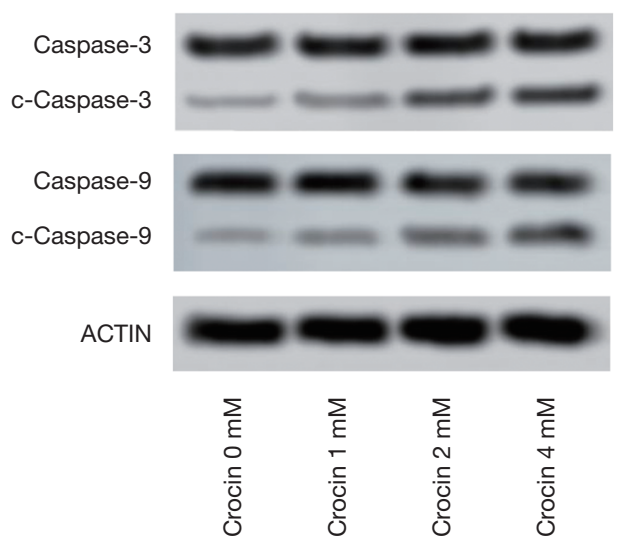

B
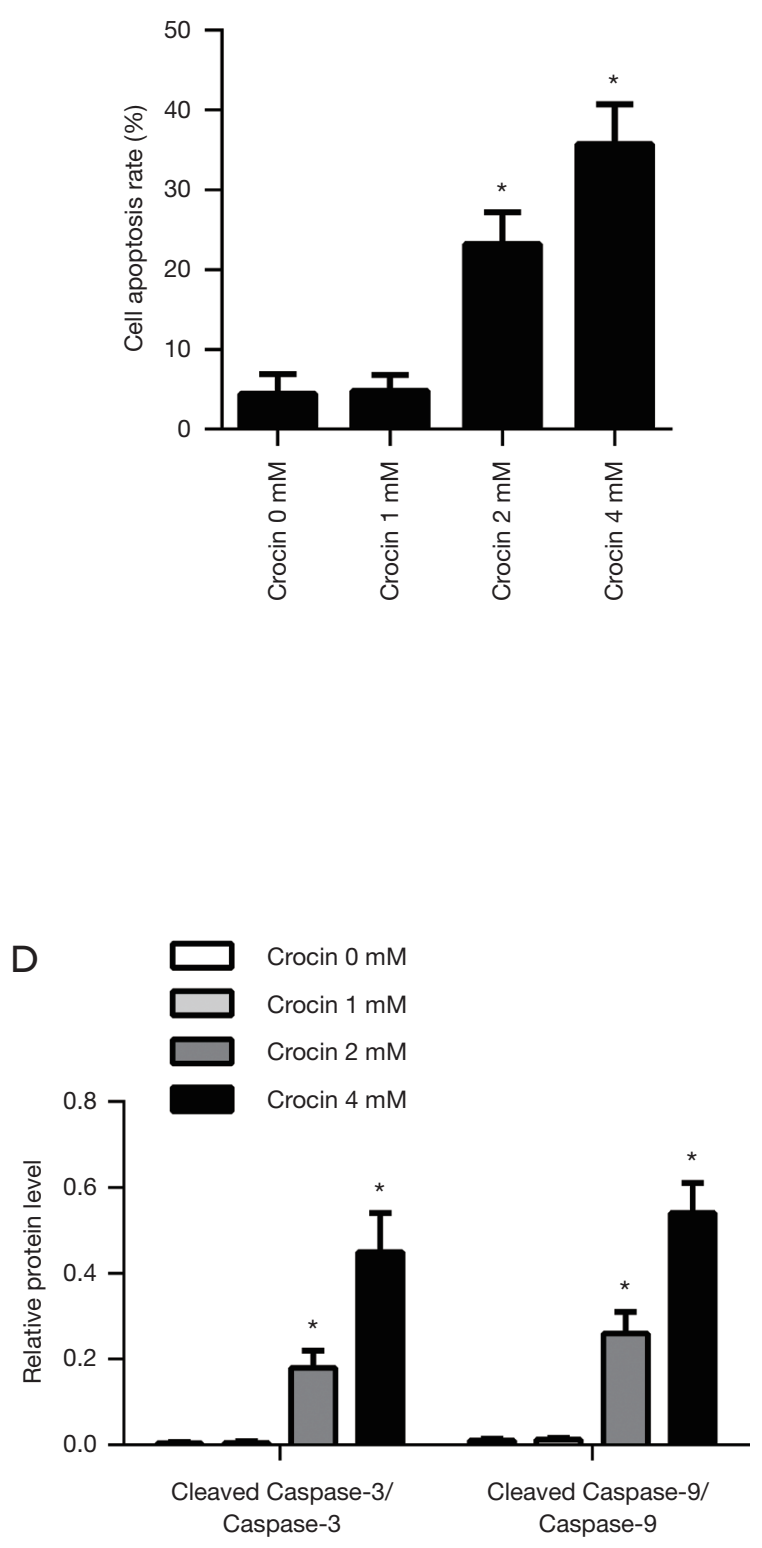

Figure 2 Apoptosis of SiHa cells treated with crocin. SiHa cells were treated with 0, 1, 2 and 4 mM of crocin for 24 hours. (A) The apoptotic SiHa cells were assessed using flow cytometry; (B) the apoptotic cell counts are shown in bar graphs; (C) markers of apoptosis were measured with western blotting; (D) the ratio of cleaved caspase-3/caspase-3 and cleaved caspase-9/caspase-9 were estimated with Image J. *, $\mathrm{P}<0.05$ versus control with $t$-test.

the development and application of crocin, such as raising blood glucose, competing receptors, or changing the in vivo distribution of drugs.

Currently, the mechanism of action for crocin in cervical cancer mainly includes the induction of apoptosis and the inhibition of proliferation (8). Crocin also suppressed spindle microtubule dynamics and reduced the cell viability of HeLa cells without significantly increasing cellular ROS 
A
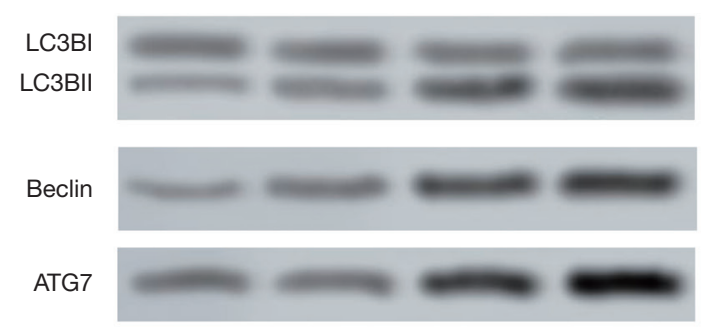

ACTIN

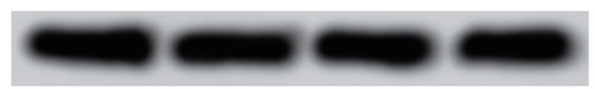

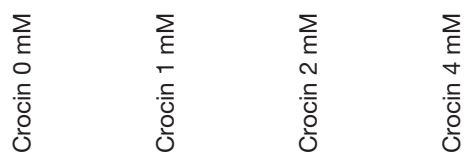

B

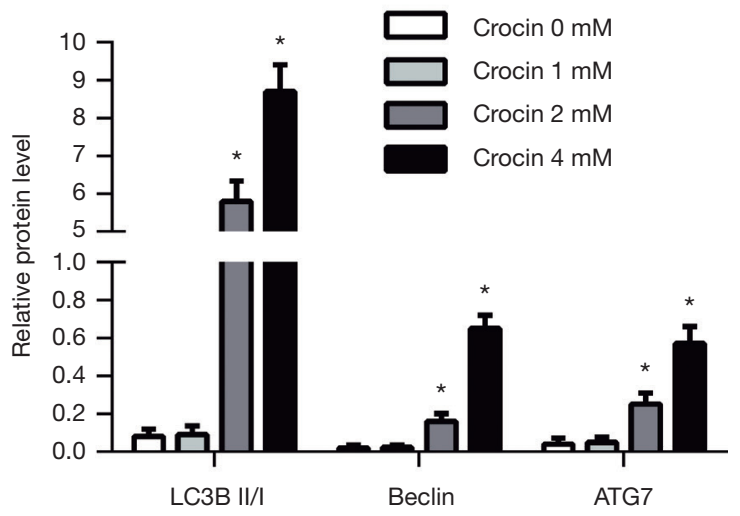

C

Crocin $0 \mathrm{mM}$

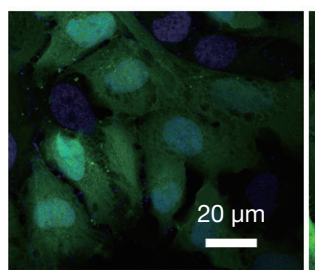

Crocin $1 \mathrm{mM}$

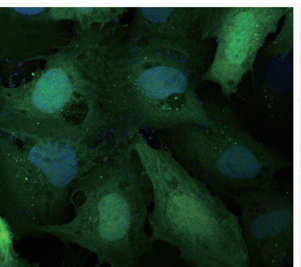

Crocin $2 \mathrm{mM}$

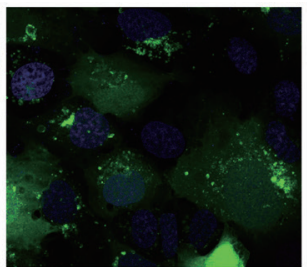

Crocin $4 \mathrm{mM}$

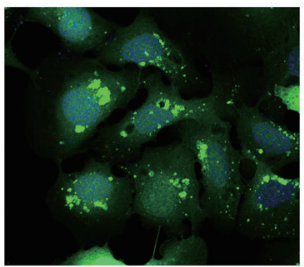

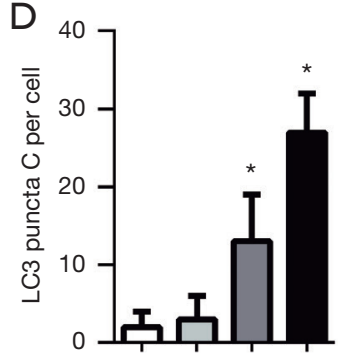

Figure 3 Autophagy of SiHa cells treated with crocin. SiHa cells were treated with 0, 1, 2 and $4 \mathrm{mM}$ of crocin for 24 hours. (A) Expression of autophagy marker proteins were measured with western blotting; (B) the relative expression of LIC II/I, Beclin1 and ATG7 were estimated with Image J; (C) LC3 puncta in SiHa cells were visualized with immunofluorescence and observed under 400× scope, green: LC3 puncta, blue: nucleus; (D) LC3 puncta per cell were evaluated with Image Pro Plus. *, $\mathrm{P}<0.05$ versus control with $t$-test.

A

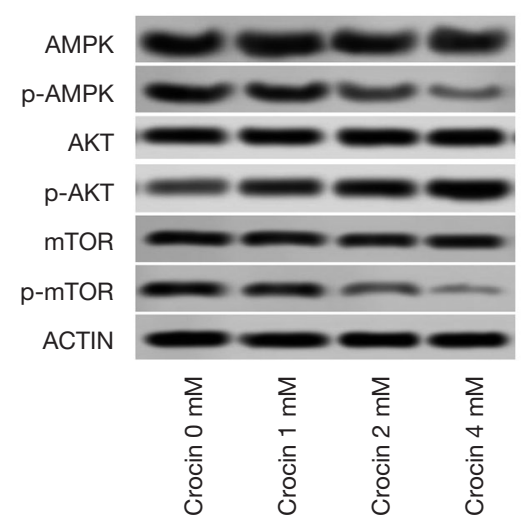

B

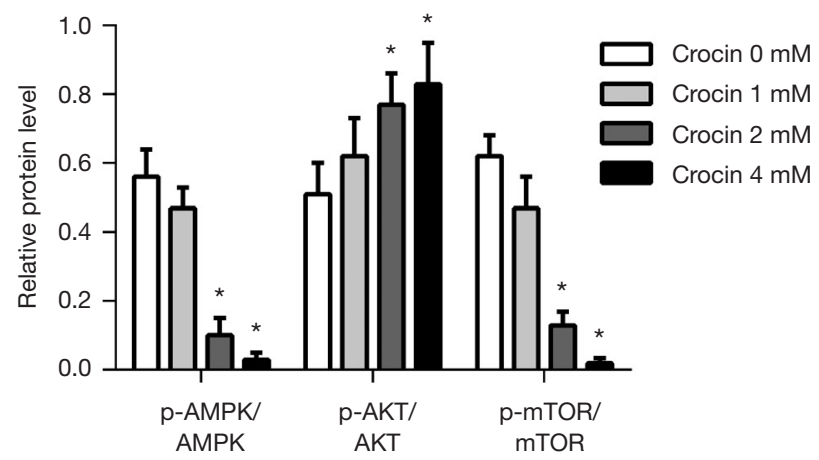

Figure 4 AMPK, AKT and mTOR expression in SiHa cells treated with crocin. SiHa cells were treated with 0,1,2 and $4 \mathrm{mM}$ of crocin for 24 hours. (A) Protein expression of AMPK, p-AMPK, AKT, p-AKT, mTOR and p-mTOR were measured with western blotting; (B) the protein expression profiles were measured with Inage $\mathrm{J}$. *, $\mathrm{P}<0.05$ versus control with $t$-test. 
A

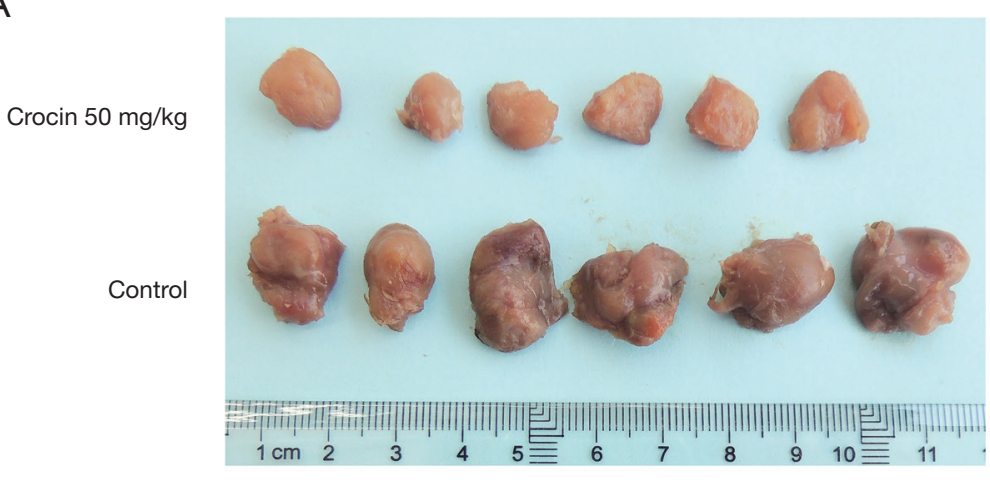

C
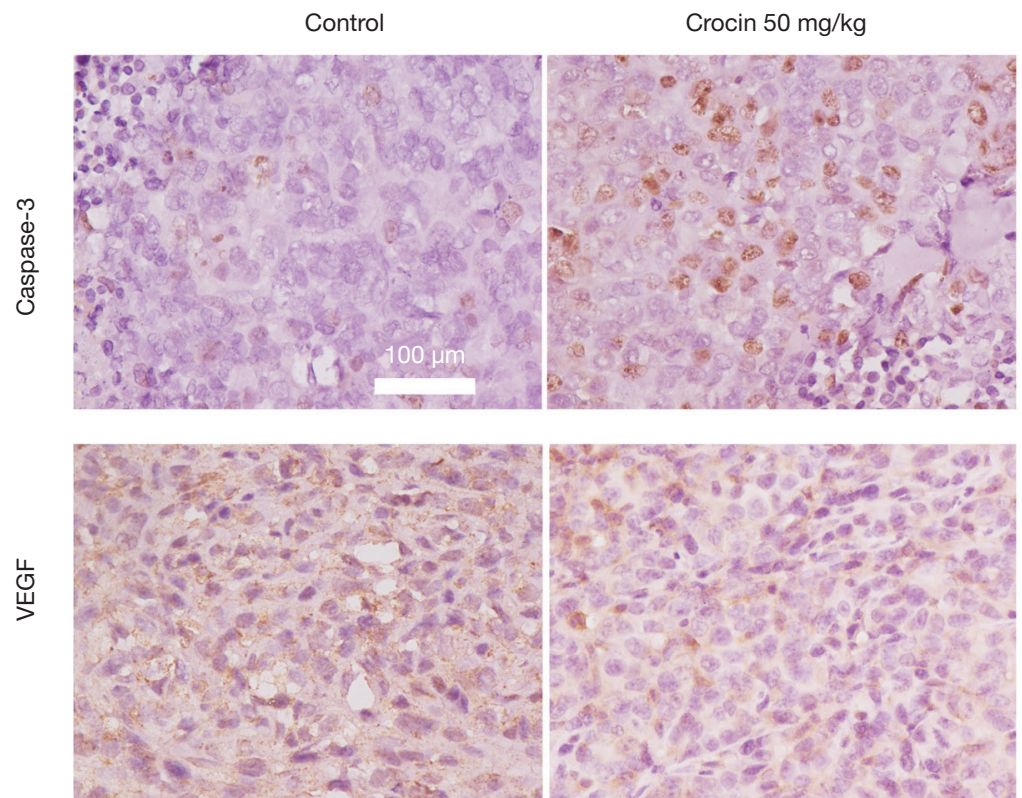

$\mathrm{F}$

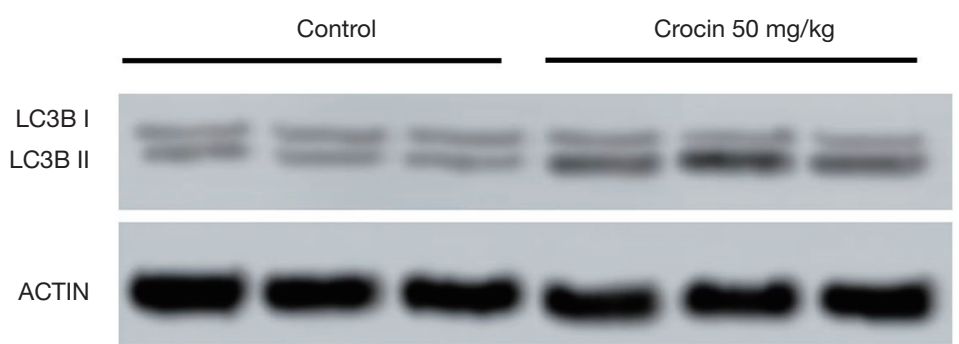

B

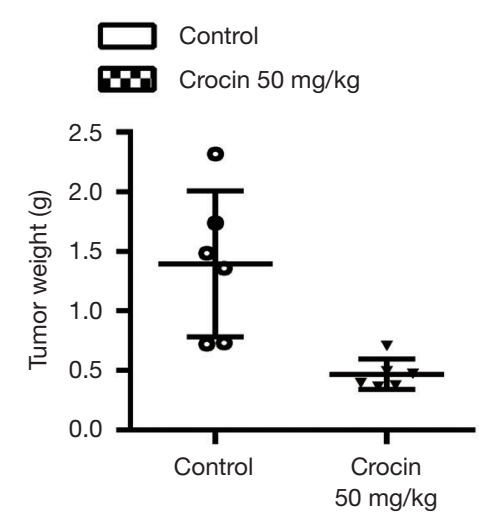

$\mathrm{D}$

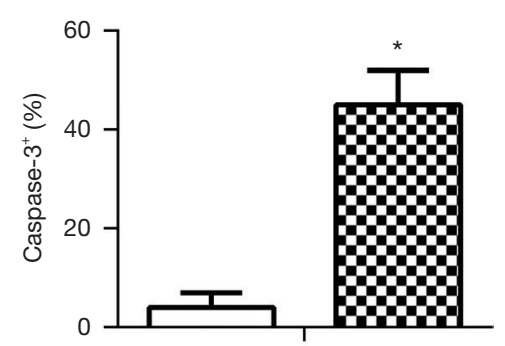

E

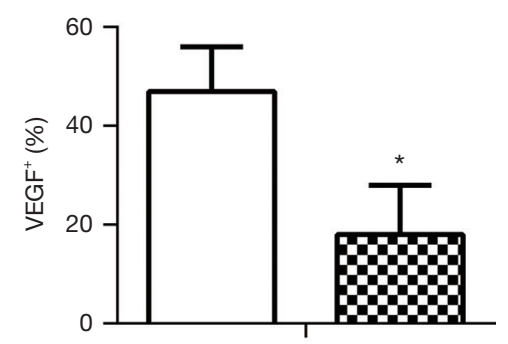

G

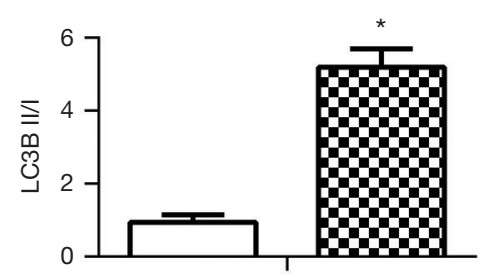

Figure 5 In vivo effects of crocin. SiHa cervical cancer cells were inoculated in female BALB/c mice. The mice were injected with crocin $(50 \mathrm{mg} / \mathrm{kg} / \mathrm{d})$ or saline for 28 days. (A) The tumors were imaged; (B) the tumors were weighed and compared; (C) immunohistochemical staining of the tumors observed under 200x scope showing caspase-3 and VEGF, violet: cell nucleus, yellow: Caspase-3 or VEGF positive signal. Caspase-3 (D) and VEGF (E) positive cells were counted; (F) LC3 expression in tumors; (G) the relative expression of LC3II/I was measured with Image J. *, $\mathrm{P}<0.05$ versus control with $t$-test. 
$(30,31)$. In the present study, we also demonstrated a dosedependent inhibition of cell proliferation. Additionally, invasive cells were reduced after crocin exposure, and apoptosis and autophagy were induced after crocin treatment. These results suggest that autophagy induced apoptosis in crocin treated $\mathrm{SiHa}$ cells. The AMPK, AKT and mTOR pathways are implicated in the regulation of autophagy and apoptosis in crocin treated cells in other tumors (12). In the present study, AMPK and mTOR were inactivated while PI3K was activated with crocin treatment. These results suggest that induced autophagy was a result of inhibited AMPK rather than PI3K.

Using a relatively high concentration, Jiang et al. showed that $125 \mathrm{mg} / \mathrm{L}$ of crocin inhibited the proliferation of HeLa cells in vitro; and $120 \mathrm{mg} / \mathrm{kg}$ of crocin in male albino rats decreased alanine transaminase (ALT), aspartate transaminase (AST), lactate dehydrogenase (LDH), alkaline phosphatase (ALP), blood urea nitrogen (BUN), creatinine, bilirubin, albumin and total protein, while increasing glucose, cholesterol, thyroglobulin (TG), and glutathione (GSH) (32). Based on these results, they argued that the adverse effects of crocin might limit its clinical application. However, crocin has long been utilized as a food colorant and additive in the food industry worldwide, which strongly supports its safety profile (33). On the other hand, less protein/peptides and more glucose or TG in serum may be of benefit in combating tumors, as the protein supply of tumors can be limited and the immune system can be activated. In this study, we observed no significant adverse effects in mice. Crocin administration significantly reduced tumor growth, and promoted autophagy and apoptosis. These results support the efficacy and safety of crocin for cancer treatment.

There are limitations to this study. There are many reported targets of crocin, and the mechanism of action for crocin in cervical cancer is complicated, but only one aspect was investigated in the current study. The complexity of crocin also raises concerns about potential adverse effects and possible interactions with other therapeutics, which remain largely unexplored.

In conclusion, we showed that crocin inhibited proliferation and invasion, and promoted autophagy and apoptosis of $\mathrm{SiHa}$ cells and in xenograft mice. We propose that the anti-tumor effects of crocin was a result of induced autophagy through AMPK and mTOR inhibition but not PI3K. Also, the results suggest that crocin could be a potential prodrug for cervical cancer treatment.

\section{Acknowledgments}

Funding: None.

\section{Footnote}

Reporting Checklist: The authors have completed the ARRIVE reporting checklist. Available at http://dx.doi. org/10.21037/atm-20-5882

Data Sharing Statement: Available at http://dx.doi. org/10.21037/atm-20-5882

Conflicts of Interest: All authors have completed the ICMJE uniform disclosure form (available at http://dx.doi. org/10.21037/atm-20-5882). The authors have no conflicts of interest to declare.

Ethical Statement: The authors are accountable for all aspects of the work in ensuring that questions related to the accuracy or integrity of any part of the work are appropriately investigated and resolved. The study was approved by the Medical Ethics Committee of West China Second Hospital, Sichuan University (No. 2014-2020). All animal experiments were approved by the Institutional Animal Care and Use Committee of Sichuan Academy of Medical Sciences \& Sichuan Provincial People's Hospital, and were performed according to institutional guidelines.

Open Access Statement: This is an Open Access article distributed in accordance with the Creative Commons Attribution-NonCommercial-NoDerivs 4.0 International License (CC BY-NC-ND 4.0), which permits the noncommercial replication and distribution of the article with the strict proviso that no changes or edits are made and the original work is properly cited (including links to both the formal publication through the relevant DOI and the license). See: https://creativecommons.org/licenses/by-nc-nd/4.0/.

\section{References}

1. Siegel RL, Miller KD, Jemal A. Cancer statistics, 2019. CA Cancer J Clin 2019;69:7-34.

2. Canfell K, Kim JJ, Brisson M, et al. Mortality impact of achieving WHO cervical cancer elimination targets: a comparative modelling analysis in 78 low-income and lower-middle-income countries. Lancet 2020;395:591-603. 
3. Xue C, Zhu D, Chen L, et al. Expression and prognostic value of PD-L1 and PD-L2 in ovarian cancer. Transl Cancer Res 2019;8:111-9.

4. Mattoscio D, Medda A, Chiocca S. Human Papilloma Virus and Autophagy. Int J Mol Sci 2018:19(6).

5. Hoshyar R, Mollaei H. A comprehensive review on anticancer mechanisms of the main carotenoid of saffron, crocin. J Pharm Pharmacol 2017;69:1419-27.

6. Moradzadeh M, Kalani MR, Avan A. The antileukemic effects of saffron (Crocus sativus L.) and its related molecular targets: A mini review. J Cell Biochem 2019;120:4732-8.

7. Mollaei H, Safaralizadeh R, Babaei E, et al. The anti-proliferative and apoptotic effects of crocin on chemosensitive and chemoresistant cervical cancer cells. Biomed Pharmacother 2017;94:307-16.

8. Yao C, Liu BB, Qian XD, et al. Crocin induces autophagic apoptosis in hepatocellular carcinoma by inhibiting Akt/ mTOR activity. OncoTargets and therapy 2018:11:2017-28.

9. Liu S, Wang H, Mu J, et al. MiRNA-211 triggers an autophagy-dependent apoptosis in cervical cancer cells: regulation of Bcl-2. Naunyn Schmiedebergs Arch Pharmacol 2020;393:359-70.

10. Rastgoo M, Hosseinzadeh H, Alavizadeh H, et al. Antitumor activity of PEGylated nanoliposomes containing crocin in mice bearing C26 colon carcinoma. Planta Med 2013;79:447-51.

11. Zhang X, Zhang X, Dang Z, et al. Cognitive Protective Mechanism of Crocin Pretreatment in Rat Submitted to Acute High-Altitude Hypoxia Exposure. Biomed Res Int 2020;2020:3409679.

12. Zeng C, Li H, Fan Z, et al. Crocin-Elicited Autophagy Rescues Myocardial Ischemia/Reperfusion Injury via Paradoxical Mechanisms. Am J Chin Med 2016;44:515-30.

13. Utada $M$, Chernyavskiy $P$, Lee $W J$, et al. Increasing risk of uterine cervical cancer among young Japanese women: Comparison of incidence trends in Japan, South Korea and Japanese-Americans between 1985 and 2012. Int J Cancer 2019;144:2144-52.

14. Johnson CA, James D, Marzan A, et al. Cervical Cancer: An Overview of Pathophysiology and Management. Semin Oncol Nurs 2019;35:166-74.

15. Naga Ch P, Gurram L, Chopra S, et al. The management of locally advanced cervical cancer. Curr Opin Oncol 2018;30:323-9.

16. Veisi A, Akbari G, Mard SA, et al. Role of crocin in several cancer cell lines: An updated review. Iran J Basic Med Sci 2020;23:3-12.
17. Luo Y, Cui S, Tang F, et al. The combination of crocin with cisplatin suppresses growth of gastric carcinoma cell line BGC-823 and promotes cell apoptosis. Pak J Pharm Sci 2017;30:1629-34.

18. Chen S, Zhao S, Wang X, et al. Crocin inhibits cell proliferation and enhances cisplatin and pemetrexed chemosensitivity in lung cancer cells. Transl Lung Cancer Res 2015;4:775-83.

19. Kawabata K, Tung NH, Shoyama Y, et al. Dietary Crocin Inhibits Colitis and Colitis-Associated Colorectal Carcinogenesis in Male ICR Mice. Evid Based Complement Alternat Med 2012;2012:820415.

20. Mir MA, Ganai SA, Mansoor S, et al. Isolation, purification and characterization of naturally derived Crocetin beta-dglucosyl ester from Crocus sativus L. against breast cancer and its binding chemistry with ER-alpha/HDAC2. Saudi J Biol Sci 2020;27:975-84.

21. Arzi L, Farahi A, Jafarzadeh N, et al. Inhibitory Effect of Crocin on Metastasis of Triple-Negative Breast Cancer by Interfering with $W n t / \beta$-Catenin Pathway in Murine Model. DNA Cell Biol 2018;37:1068-75.

22. Chen SS, Gu Y, Lu F, et al. Antiangiogenic effect of crocin on breast cancer cell MDA-MB-231. J Thorac Dis 2019;11:4464-73.

23. Hoshyar R, Bathaie SZ, Kyani A, et al. Is there any interaction between telomeric DNA structures, G-quadruplex and I-motif, with saffron active metabolites? Nucleosides Nucleotides Nucleic Acids 2012;31:801-12.

24. Aanouz I, Belhassan A, El-Khatabi K, et al. Moroccan Medicinal plants as inhibitors against SARS-CoV-2 main protease: Computational investigations. J Biomol Struct Dyn 2020:1-9.

25. Patil S, Sistla S, Jadhav J. Interaction of small molecules with human tyrosinase: A surface plasmon resonance and molecular docking study. Int J Biol Macromol 2016;92:1123-9.

26. Salem AA, Lotfy M, Amin A, et al. Characterization of human serum albumin's interactions with safranal and crocin using multi-spectroscopic and molecular docking techniques. Biochem Biophys Rep 2019;20:100670.

27. Luo S, Gill H, Feltis B, et al. The Effects of a WeightLoss Herbal Formula RCM-107 and Its Eight Individual Ingredients on Glucagon-Like Peptide-1 Secretion-An In Vitro and In Silico Study. Int J Mol Sci 2020;21:2854.

28. Hashemi SA, Bathaie SZ, Mohagheghi MA. Interaction of saffron carotenoids with catalase: in vitro, in vivo and molecular docking studies. J Biomol Struct Dyn 2020;38:3916-26. 
29. Khedr LH, Rahmo RM, Farag DB, et al. Crocin attenuates cisplatin-induced hepatotoxicity via TLR4/NF-кBp50 signaling and BAMBI modulation of TGF- $\beta$ activity: Involvement of miRNA-9 and miRNA-29. Food Chem Toxicol 2020;140:111307.

30. Kim SH, Lee JM, Kim SC, et al. Proposed cytotoxic mechanisms of the saffron carotenoids crocin and crocetin on cancer cell lines. Biochem Cell Biol 2014;92:105-11.

31. Sawant AV, Srivastava S, Prassanawar SS, et al. Crocin, a carotenoid, suppresses spindle microtubule dynamics and activates the mitotic checkpoint by binding to tubulin.

Cite this article as: Zhang J, Yang S, Wang K, Huang Y, Yang N, Yang Z, Zheng Z, Wang Y. Crocin induces autophagic cell death and inhibits cell invasion of cervical cancer SiHa cells through activation of PI3K/AKT. Ann Transl Med 2020;8(18):1180. doi: 10.21037/atm-20-5882
Biochem Pharmacol 2019;163:32-45.

32. Jiang Z, Gu M, Liu J, et al. Anticancer activity of crocin against cervical carcinoma (HeLa cells): Bioassessment and toxicity evaluation of crocin in male albino rats. J Photochem Photobiol B 2018;180:118-24.

33. Mohan S, Hemachandran H, Sneha P, et al. Structural insights into the binding mode and conformational changes of BSA induced by bixin and crocin. J Biomol Struct Dyn 2018;36:2085-98.

(English Language Editor: C. Belazar-Maseh) 